Original article

\title{
Variability of salivary metabolite levels in patients with Sjögren's syndrome
}

\author{
Maria Herrala ${ }^{1,2,3)}$, Jopi J. W. Mikkonen ${ }^{4)}$, Paula Pesonen ${ }^{5)}$, Reijo Lappalainen ${ }^{4)}$, Leo Tjäderhane ${ }^{3,6}$, Raija K. Niemelä7), \\ Hubertus Seitsalo ${ }^{8)}$, Tuula Salo, ${ }^{3,6)}$, Sami Myllymaa ${ }^{4}$, and Arja M. Kullaa ${ }^{1,2,9)}$
}

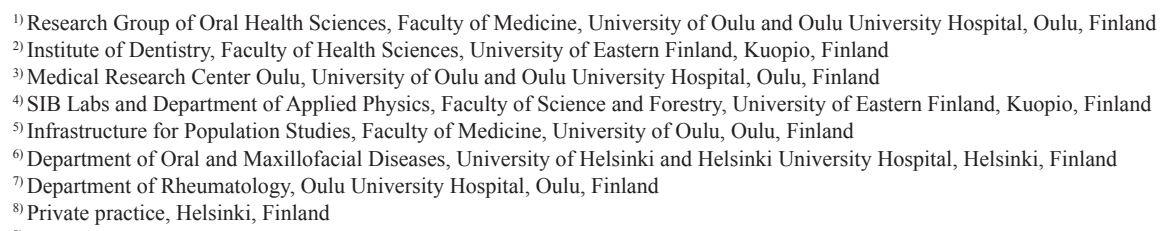

9) Educational Dental Clinic, Kuopio University Hospital, Kuopio, Finland

(Received December 4, 2019; Accepted April 21, 2020)

\begin{abstract}
Purpose: To investigate inter- and intra-individual variation in the levels and outputs (concentration multiplied by salivary flow rate) of salivary metabolites in patients with primary Sjögren's syndrome (pSS).

Methods: A total of 56 samples of stimulated saliva were collected from 14 female pSS patients during four laboratory visits within 20 weeks and analyzed using proton nuclear magnetic resonance spectroscopy. Single saliva samples from each of 15 controls were also analyzed.

Results: Among 21 quantified metabolites, choline was significantly elevated in the pSS patients at each time point $(P \leq 0.015)$, taurine at the last three time points $(P \leq 0.013)$, alanine at the last two time points $(P \leq$ $0.007)$ and glycine at the last time point $(P=0.005)$. Inter-individual variation in metabolite concentrations was generally larger among the patients than among the controls, and significantly large variations were observed for glycine ( $P \leq 0.007$, all time points), choline ( $P \leq 0.033$, three last time points) and alanine $(P=0.028$, baseline). Metabolite output analysis showed that choline had the lowest intra-patient variation.

Conclusion: In spite of considerable intra- and inter-individual variation, levels and outputs of specific metabolites in patients with pSS differ from those in controls, and may be potentially applicable as new biological markers for monitoring of the response to treatment.
\end{abstract}

Keywords; biological markers, hyposalivation, metabolomics, oral diagnosis, proton magnetic resonance spectroscopy

\section{Introduction}

Sjögren's syndrome (SS) is a systemic and slowly progressive autoimmune disease affecting mainly the salivary and lacrimal glands, although other exocrine glands may also be affected. There are two forms of SS: primary (pSS) and secondary (sSS). pSS is a discrete disease with all the typical symptoms, whereas sSS is associated with other forms of autoimmune disease that may constitute the primary diagnosis, for example systemic lupus erythematosus (SLE) [1]. There is evidence to suggest that the defective secretory processes that characterize SS are due to dysfunction of neural regulation [2]. Although the environmental factors responsible for SS development remain unknown, a recent study [3] has suggested that dysbiosis may play an important role. Typically, SS patients may suffer common symptoms of SS such as severe dry mouth and eyes for many years before being definitively diagnosed [4]. Primary SS may exhibit various clinical phenotypes with diverse outcomes. Patients with specific

Correspondence to Dr. Maria Herrala, Research Group of Oral Health Sciences, Faculty of Medicine, University of Oulu, and Oulu University Hospital, Oulu, Finland, P.O. BOX 5281, Oulu FI 90014, Finland

Fax: +358-8-537-5560 E-mail: marherra@student.oulu.fi

J-STAGE Advance Publication: October 29, 2020

Color figures can be viewed in the online issue at J-STAGE.

doi.org/10.2334/josnusd.19-0504

DN/JST.JSTAGE/josnusd/19-0504 clinical symptoms such as purpura, peripheral nervous system involvement and salivary gland enlargement have an increased risk of lymphoma $[5,6]$. Therefore, it is important to develop new methods for earlier diagnosis of SS and to monitor patients' conditions, disease development and treatment responses. Currently there is no specific laboratory test for diagnosis of pSS.

Salivary metabolomics, the global analysis of low-molecular-weight metabolites, provides an alternative to the traditional single-biomarker approach for assessment of oral diseases. Metabolomics allows quantitative measurement of the oral defense system's multi-parametric metabolic responses to pathophysiological stimuli by revealing dynamic changes in salivary metabolites. In diagnostics based on salivary metabolites, a combination pattern of several biomarkers rather than only one may define a specific disease [7]. The specific metabolite profile mirrors the current state of any given individual's health, and can be useful for monitoring of patients with various diseases.

High-resolution nuclear magnetic resonance (NMR) spectroscopy is a powerful and reproducible metabolic profiling technique, and when combined with advanced multivariate analysis methodologies it has several advantages over classical biochemical assays [8]. Recently, techniques such as gas chromatography mass spectrometry (GC-MS), liquid-chromatography mass spectrometry (LC-MS) and two-dimensional gel electrophoresis have been frequently used to analyze saliva samples. However, the use of NMR spectroscopy in saliva research has been very limited $[9,10]$. Recently, Gardner et al. have suggested that a protocol 'gold standard' should be established for preparation of saliva samples for NMR analysis [11].

A number of previous studies have investigated the salivary metabolic profile of patients with pSS. So far, a total of 24 metabolites have been identified in samples of stimulated saliva [12], and some of them, including choline, butyrate, proline, taurine, alanine, phenylalanine and glycine, have been shown to have significantly higher concentrations in saliva from pSS patients than in that from controls. In particular, the concentrations of choline and taurine have been shown to be associated with changes in salivary flow rate [12].

The factors that influence the metabolic composition of saliva and contribute to variations in metabolic profiles include genetics, sex, age, diurnal cycle, diet, hormone concentrations, drug effects, stress, oral health, oral microflora and oral hygiene [13]. Therefore, the metabolic profile of saliva shows considerable inter- and intra-individual variation and this can shed light on the physiological factors that might contribute to it.

The aim of the present study was to assess inter- and intra-individual variation of salivary metabolic profiles in patients with pSS in comparison with the salivary metabolome of control subjects using proton nuclear magnetic resonance ( $\left.{ }^{1} \mathrm{H}-\mathrm{NMR}\right)$ spectroscopy. The working hypothesis was that there would be differences in the inter- and intra-individual metabolomic profiles of pSS patients and that these differences would be detectable using quantitative ${ }^{1} \mathrm{H}-\mathrm{NMR}$ spectroscopy. 
Table 1 Comparison of 21 salivary metabolite concentrations in pSS patients in the four time points of the study

\begin{tabular}{|c|c|c|c|c|c|c|c|c|c|c|c|c|}
\hline \multicolumn{13}{|c|}{ Concentration $(\mu \mathrm{M})$} \\
\hline Metabolite & At the baseline & After 1 week & $n$ & $P$ value & At the baseline & After 10 weeks & $n$ & $P$ value & At the baseline & After 20 weeks & $n$ & $P$ value \\
\hline Acetate & $907.6(529.1-2318.6)^{b}$ & $1349.7(322.5-2712.3)^{b}$ & 9 & 0.139 & $1079.1 \pm 562.6^{\mathrm{a}}$ & $1541.0 \pm 515.3^{\mathrm{a}}$ & 10 & 0.108 & $1079.1 \pm 562.6^{a}$ & $1593.2 \pm 671.2^{\mathrm{a}}$ & 10 & $0.036^{*}$ \\
\hline Alanine & $14.4(2.0-54.3)^{\mathrm{b}}$ & $19.3(4.8-33.7)^{\mathrm{b}}$ & 9 & 0.859 & $12.1(2.0-54.3)^{\mathrm{b}}$ & $19.0(13.5-43.5)^{\mathrm{b}}$ & 10 & 0.575 & $12.1(2.0-54.3)^{\mathrm{b}}$ & $28.0(11.4-42.2)^{\mathrm{b}}$ & 10 & 0.114 \\
\hline Butanol & $4.7(1.3-23-4)^{\mathrm{b}}$ & $8.1(4.3-44.7)^{\mathrm{b}}$ & 9 & $0.008^{*}$ & $5.0(1.3-23.4)^{b}$ & $14.1(5.3-50.2)^{b}$ & 10 & 0.074 & $7.9 \pm 7.6^{\mathrm{a}}$ & $23.0 \pm 9.2^{\mathrm{a}}$ & 10 & $<0.001^{* * *}$ \\
\hline Butyrate & $9.0(3.2-51.0)^{\mathrm{b}}$ & $14,9(10.1-99.6)^{b}$ & 9 & $0.028 *$ & $14.5 \pm 14.0^{\mathrm{a}}$ & $36.9 \pm 16.9^{\mathrm{a}}$ & 10 & $0.005^{* *}$ & $9.8(3.2-51.0)^{\mathrm{b}}$ & $42.1(16.6-71.4)^{b}$ & 10 & $0.005^{* *}$ \\
\hline Choline & $6.2(3.5-10.4)^{b}$ & $8.9(2.1-20.2)^{\mathrm{b}}$ & 9 & 0.173 & $6.6 \pm 2.3^{\mathrm{a}}$ & $9.5 \pm 3.5^{\mathrm{a}}$ & 10 & $0.022 *$ & $6.5(3.5-10.4)^{\mathrm{b}}$ & $9.9(4.5-22.3)^{\mathrm{b}}$ & 10 & $0.025^{*}$ \\
\hline Citrate & $16.7(8.6-27.6)^{\mathrm{b}}$ & $14.9(5.9-36.4)^{\mathrm{b}}$ & 8 & 0.674 & $13.7(8.6-27.6)^{\mathrm{b}}$ & $16.1(5.2-21.6)^{\mathrm{b}}$ & 9 & 0.767 & $16.4 \pm 6.2^{\mathrm{a}}$ & $16.6 \pm 12.7^{\mathrm{a}}$ & 9 & 0.952 \\
\hline Ethanol & $11.5 \pm 3.3^{\mathrm{a}}$ & $20.7 \pm 9.7^{\mathrm{a}}$ & 7 & 0.085 & $12.3(5.8-16.1)^{\mathrm{b}}$ & $14.3(11.9-37.0)^{b}$ & 10 & 0.139 & $12.3(5.8-16.1)^{\mathrm{b}}$ & $28.9(22.8-75.0)^{\mathrm{b}}$ & 10 & $0.005^{* *}$ \\
\hline Formate & $50.8(19.6-89.7)^{b}$ & $43.1(3.9-178.5)^{b}$ & 9 & 0.767 & $54.7 \pm 23.5^{\mathrm{a}}$ & $126.6 \pm 96.8^{\mathrm{a}}$ & 10 & 0.052 & $49.9(19.6-89.7)^{b}$ & $82.4(11.0-536.9)^{\mathrm{b}}$ & 10 & $0.022 *$ \\
\hline Fucose & $25.4(7.1-161-2)^{b}$ & $37.4(6.8-87.3)^{b}$ & 9 & 0.441 & $35.6(7.1-161.2)^{b}$ & $40.5(8.1-150.9)^{b}$ & 10 & 0.878 & $35.6(7.1-161.2)^{b}$ & $56.3(6.8-197.2)^{\mathrm{b}}$ & 10 & 0.959 \\
\hline Glycine & $66.6(13.3-232.1)^{\mathrm{b}}$ & $68.5(17.1-201.7)^{b}$ & 9 & 0.441 & $95.3 \pm 77.0^{\mathrm{a}}$ & $101.1 \pm 62.3^{\mathrm{a}}$ & 10 & 0.787 & $95.3 \pm 76.9^{\mathrm{a}}$ & $148.6 \pm 114.7^{\mathrm{a}}$ & 10 & $0.044 *$ \\
\hline Lactate & $261.3(142.6-527.2)^{\mathrm{b}}$ & $218.6(40.1-750.4)^{b}$ & 9 & 0.775 & $258.5 \pm 121.6^{\mathrm{a}}$ & $258.9 \pm 129.8^{\mathrm{a}}$ & 10 & 0.994 & $230.2(142.6-527.2)^{\mathrm{b}}$ & $237.7(25.7-752.1)^{\mathrm{b}}$ & 10 & 0.575 \\
\hline Methanol & $30.6 \pm 15.1^{\mathrm{a}}$ & $24.6 \pm 8.6^{\mathrm{a}}$ & 9 & 0.406 & $30.8 \pm 14.2^{\mathrm{a}}$ & $23.5 \pm 16.6^{\mathrm{a}}$ & 10 & 0.341 & $30.8 \pm 14.2^{\mathrm{a}}$ & $25.0 \pm 14.2^{\mathrm{a}}$ & 10 & 0.252 \\
\hline Methylamine & $1.7 \pm 0.8^{\mathrm{a}}$ & $2.7 \pm 1.7^{\mathrm{a}}$ & 7 & 0.097 & $1.8 \pm 0.7^{\mathrm{a}}$ & $2.2 \pm 1.1^{\mathrm{a}}$ & 8 & 0.194 & $1,7(0.9-2.8)^{\mathrm{b}}$ & $2.2(1.0-4.0)^{\mathrm{b}}$ & 8 & 0.458 \\
\hline Phenylalanine & $10.7(5.9-34.6)^{\mathrm{b}}$ & $12.8(3.0-36.1)^{\mathrm{b}}$ & 9 & 0.953 & $15.1 \pm 10.3^{\mathrm{a}}$ & $13.7 \pm 6.5^{\mathrm{a}}$ & 10 & 0.734 & $10.0(5.9-34.6)^{\mathrm{b}}$ & $17.7(7.3-48.7)^{\mathrm{b}}$ & 10 & 0.114 \\
\hline Proline & $128.5 \pm 123.5^{\mathrm{a}}$ & $70.0 \pm 44.14^{\mathrm{a}}$ & 8 & 0.178 & $121.9 \pm 117.2^{\mathrm{a}}$ & $76.8 \pm 70.7^{\mathrm{a}}$ & 9 & 0.204 & $121.9 \pm 117.2^{\mathrm{a}}$ & $109.6 \pm 82.9^{\mathrm{a}}$ & 9 & 0.551 \\
\hline Propane & $179.2(107.2-411.1)^{\mathrm{b}}$ & $279.3(44.9-700.9)^{\mathrm{b}}$ & 9 & 0.086 & $185.0(107.2-411.1)^{b}$ & $271.0(123.5-750.8)^{b}$ & 10 & 0.169 & $185(107.2-411.1)^{b}$ & $316.5(68.8-779.5)^{\mathrm{b}}$ & 10 & $0.037^{*}$ \\
\hline Pyruvate & $12.5(7.5-23.4)^{\mathrm{b}}$ & $12.6(3.3-25.1)^{\mathrm{b}}$ & 9 & 0.953 & $13.7 \pm 4.7^{\mathrm{a}}$ & $19.4 \pm 8.3^{\mathrm{a}}$ & 10 & 0.080 & $13.1(7.5-23.4)^{b}$ & $17.8(5.1-40.9)^{\mathrm{b}}$ & 10 & 0.074 \\
\hline Succinate & $18.5 \pm 7.5^{\mathrm{a}}$ & $29.8 \pm 20.8^{\mathrm{a}}$ & 9 & 0.114 & $17.4 \pm 8.0^{\mathrm{a}}$ & $31.3 \pm 16.6^{\mathrm{a}}$ & 10 & $0.023 *$ & $16.5(7.0-29.8)^{\mathrm{b}}$ & $25.7(6.5-142.6)^{b}$ & 10 & $0.007^{*}$ \\
\hline Taurine & $51.6 \pm 18.6^{\mathrm{a}}$ & $89.9 \pm 41.1^{\mathrm{a}}$ & 9 & $0.014 *$ & $51.0 \pm 17.6^{\mathrm{a}}$ & $107.3 \pm 46.2^{\mathrm{a}}$ & 10 & $0.001 * *$ & $51.0 \pm 17.6^{\mathrm{a}}$ & $99.2 \pm 34.4^{\mathrm{a}}$ & 10 & $<0.001^{* * *}$ \\
\hline Tyrosine & $17.6(7.5-42.6)^{\mathrm{b}}$ & $15.6(3.7-57.0)^{\mathrm{b}}$ & 9 & 0.859 & $16.8(7.5-42.6)^{\mathrm{b}}$ & $21.4(4.2-32.4)^{\mathrm{b}}$ & 10 & 0.799 & $21.3 \pm 13.6^{\mathrm{a}}$ & $30.6 \pm 21.2^{\mathrm{a}}$ & 10 & 0.160 \\
\hline 1,2-propanediol & $20.4 \pm 10.5^{\mathrm{a}}$ & $20.0 \pm 7.9^{\mathrm{a}}$ & 9 & 0.900 & $19.8 \pm 10.1^{\mathrm{a}}$ & $22.0 \pm 10.8^{\mathrm{a}}$ & 10 & 0.644 & $19.8 \pm 10.1^{\mathrm{a}}$ & $21.1 \pm 8.2^{\mathrm{a}}$ & 10 & 0.675 \\
\hline
\end{tabular}

A paired samples $t$-test [mean \pm standard deviation (a)] was used to compare baseline to three time points and if the differences between baseline and the time points were not normally distributed Wilcoxon test [median (minimummaximum) (b)] was used. In comparisons of the baseline and a single time point there were just those patients who have been involved in both steps. ${ }^{*} P<0.05 ; * * P<0.01 ; * * * P<0.001$

\section{Materials and Methods}

The present study was conducted according to the guidelines of the Declaration of Helsinki and was approved by the Oulu University Hospital Ethical Committee (EETTMK: 116/2000 and 36/2012). All participants were fully informed and signed a written consent form. Twenty-two pSS patients in the Oulu area had been diagnosed as having SS at the Department of Rheumatology, Oulu University Hospital, Oulu, Finland by the author (R.N.) in accordance with the classification criteria proposed by the European Community [14]. The patient inclusion and exclusion criteria have been described previously in detail by Niemelä et al. [15]. Three SS patients left the study at an early stage. The remaining patients underwent an oral and dental examination performed by a clinical dentist (H.S.). One patient had gingivitis and some of the patients had secondary caries or cervical primary caries lesions. All of these issues were treated before saliva collection. Saliva samples were collected in the Training Clinic, Department of Dentistry, University of Oulu by the author (H.S.), and were the same samples as those used in the studies by Seitsalo et al. and Mikkonen et al. $[16,12]$. Patients with a smoking habit and with oral or systemic diseases other than pSS were excluded. The patient group consisted of 19 pSS female patients aged between 28 and 68 years (mean age 48.6 years).

\section{Saliva collection}

A total of 56 saliva samples were collected from the pSS patients at four time points: at the baseline, and after 1, 10 and 20 weeks. The control group consisted of 15 healthy, non-smoking females aged between 28 and 68 years (mean age 49.8 years). Only one saliva sample was taken from each of these controls. None of the control subjects had any chronic diseases or were receiving any treatment that would affect the saliva test results. Saliva samples were collected over four laboratory visits (at the baseline, and after 1 week, 10 weeks and 20 weeks) using established protocols previously described by Navazesh [17]. All saliva samples were collected in the morning between $10 \mathrm{am}$ and $12 \mathrm{am}$, and at least $1 \mathrm{~h}$ after eating and drinking. Stimulated whole mouth saliva (SWMS) was collected by chewing paraffin wax (Orion Diagnostica, Espoo, Finland; $1 \mathrm{~g}$ ) for $30 \mathrm{~s}$ and all produced saliva was collected over a 5 -min period. Saliva flow rates $(\mathrm{mL} / \mathrm{min})$ were calculated immediately after collection. The saliva samples were put on ice and transported to the laboratory. They were then centrifuged at $3,000 \times g\left(20 \mathrm{~min}\right.$ at $\left.4^{\circ} \mathrm{C}\right)$ and the supernatants were frozen at $-20^{\circ} \mathrm{C}$.

\section{Sample preparation and NMR measurements}

Sample preparation and NMR spectral acquisition were performed using a previously described protocol [12]. Briefly, saliva samples were thawed and $450 \mu \mathrm{L}$ of each aliquot was carefully mixed with $50 \mu \mathrm{L}$ of NMRbuffer (1.5 M KH $\mathrm{MHO}_{4}, 2 \mathrm{mM} \mathrm{NaN}_{3}, 5.8 \mathrm{mM}$ sodium 3-(trimethylsilyl) propionate-2,2,3,3- $\mathrm{d}_{4}, \mathrm{D}_{2} \mathrm{O}, \mathrm{pH}$ 7.4). For removal of debris, the mixture was centrifuged at $10,000 \times g$ for $5 \mathrm{~min}$ at $4^{\circ} \mathrm{C}$, after which the supernatant was transferred to a 5-mm (outer diameter) NMR tube. The samples were subjected to ${ }^{1} \mathrm{H}-\mathrm{NMR}$ spectroscopy on a Bruker Avance III HD spectrometer (Bruker Biospin GmbH Rheinstetten, Karlsruhe, Germany) operating at $600.20 \mathrm{MHz}$ at $25^{\circ} \mathrm{C}$. The spectral acquisition was controlled with TopSpin 3.2 (Bruker) software. The automated Topshim routine (Bruker) was used in shimming. NMR spectra were acquired using a $\mathrm{T}_{2}$-relaxation-filtered pulse sequence that suppressed most of the broad macromolecule signals. A Bruker cpmg1d (Carr-Purcell-Meiboom-Gill) pulse sequence was used to suppress the water peak.

\section{Data processing}

NMR spectral processing was done using a previously described protocol [12]. Briefly, the raw NMR spectra were manually corrected for phase using TopSpin 3.2 software. Before Fourier transformation, the free induction decays were multiplied by an exponential window function corresponding to a $1.0-\mathrm{Hz}$ line broadening factor. In total, 22 metabolites were identified by reference to previous publications $[18,19]$ and a freely available Saliva Metabolome electronic database (www.salivametabolome.ca). The PERCH NMR software (PERCH Solutions Ltd, Kuopio, Finland) was used for quantification of reliably assigned salivary metabolites. The constrained total-line-shape fitting approach allowed accurate quantification of the assigned metabolites, even if the baseline was not linear or there were overlapping signals [20]. Metabolites were quantified based on the ratio of the integral of a known assignment relative to the integral of the internal reference compound (trimethylsilyl propionate, TSP) peak with a known concentration. Proton ratios of the metabolite peak to the TSP peak were calculated, and then multiplied by the dilution factor of the sample caused by addition of the standard TSP solution. The final concentrations are reported as $\mu \mathrm{mol} / \mathrm{L}$ in saliva. The quantification limit of the platform is below $1 \mu \mathrm{mol} / \mathrm{L}$, but the exact limit depends on the metabolite characteristics.

\section{Statistical analyses}

Metabolite concentrations are expressed as mean \pm standard deviation (SD) in Table 1. The Shapiro-Wilk test, the values of kurtosis, skewness, and boxplots were used to analyze the data for normality distribution. Mann-Whitney $U$-test was used to compare stimulated salivary metabolite baseline concentrations between pSS patients and the healthy control group. Levene's test was used to compare metabolite concentration variances of pSS patients at four time points to variance of the control group at 
Table 2 Chronological data for $10 \mathrm{pSS}$ patients including the coefficient of variation (CV) values representing intra-individual variation in selected metabolite (alanine, choline, glycine, taurine) outputs

\begin{tabular}{|c|c|c|c|c|c|c|c|}
\hline Patient number & ATS & ATD & SBD (years) & Alanine & Choline & Glycine & Taurine \\
\hline 1. & 55 & 46 & $>5$ & 47.0 & 21.7 & 63.4 & 50.3 \\
\hline 2. & 40 & 38 & 1 & 62.7 & 34.6 & 68.0 & 40.1 \\
\hline 3. & 63 & 59 & -10 & 29.0 & 83.5 & 99.0 & 29.5 \\
\hline 5. & 55 & 49 & $>100$ & 84.4 & 29.7 & 32.3 & 36.7 \\
\hline 7. & 70 & 65 & $>10$ & 35.9 & 39.3 & 29.7 & 33.0 \\
\hline 9. & 50 & 40 & $>10$ & 36.3 & 16.6 & 35.5 & 43.9 \\
\hline 10. & 63 & 56 & 3 & 38.3 & 23.6 & 22.4 & 37.1 \\
\hline 15. & 59 & 49 & 9 & 65.5 & 31.1 & 53.3 & 46.8 \\
\hline 18. & 61 & 54 & unknown & 37.9 & 20.2 & 47.8 & 34.2 \\
\hline 19. & 30 & 28 & 3 & 47.9 & 18.1 & 29.9 & 21.5 \\
\hline
\end{tabular}
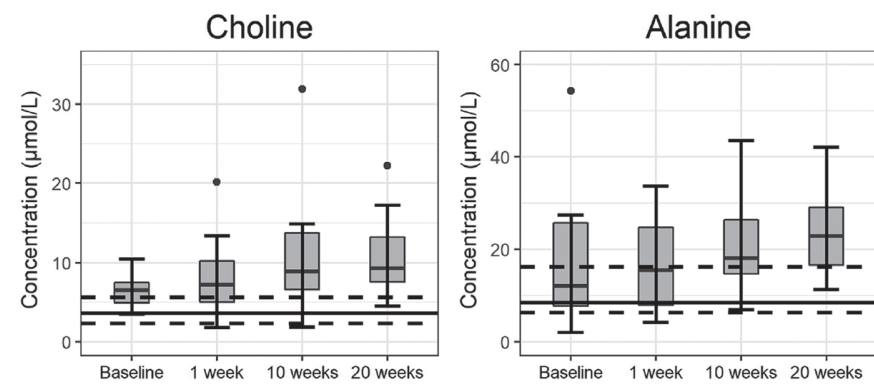

Taurine
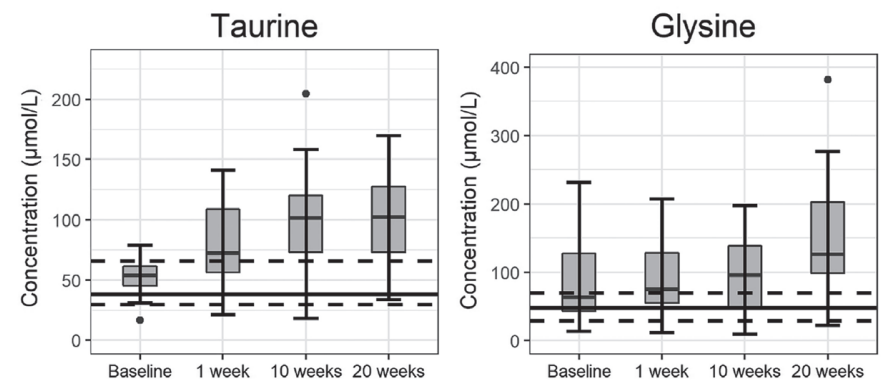

Fig. 1 Box and whisker plots illustrating variations in choline, taurine, alanine and glycine concentrations in saliva collected from pSS patients four times during 20 weeks. The median is depicted by a horizontal line in the middle portion of the box. The bottom and top boundaries of the boxes represent the lower and upper quartiles, respectively, and whiskers represent the 95 th and 5 th percentiles. Healthy controls $(n=15)$ are illustrated as horizontal lines. A continuous line represents the median and the dashed lines represent upper and lower quartiles.

the baseline. A paired samples $t$-test was used to compare baseline values to the values at three later time points, and if the differences between the baseline and the timepoints were not normally distributed, the Wilcoxon test was used. The distributions of pSS salivary metabolite concentrations with the medians, lower and upper quartiles were illustrated with box and whisker plots. The medians, lower and upper quartiles of the healthy controls were added to the plots with horizontal lines.

Intra-individual variation was determined by deriving the metabolite output $(\mu \mathrm{mol} / \mathrm{min})$ as metabolite concentration $(\mu \mathrm{mol} / \mathrm{L})$ multiplied by salivary flow rate $(\mathrm{mL} / \mathrm{min})$, and this parameter was normalized by dividing subsequent output values ( 1 week, 10 weeks, 20 weeks) by the baseline output value. Intra-individual changes in alanine, glycine, choline, and taurine in the five pSS patients at four different time points were illustrated with line diagrams. The coefficient of variation $(\mathrm{CV}=\mathrm{SD} /$ mean $)$ was used to estimate intra-individual variability of metabolite output values.

The box and whisker plots and line diagrams were executed with Rstudio (Version 1.0.143 https://www.rstudio.org). SPSS software, version 24.0 (SPSS Inc., Chicago, IL, USA) was used for all statistical analyses. Statistical significance was set at a level of $P<0.05$.

\section{Results}

Among the 19 patients enrolled, two left the study before the saliva samples were collected and two dropped out while the research was being conducted or did not produce enough saliva for NMR analysis. Furthermore, one patient's saliva samples were contaminated with lipstick and were therefore excluded from analysis. The final number of patients was thus 14. Table 2 represents the time between SS patients experiencing SSrelated symptoms and SS diagnosis, patient age at the time of diagnosis, and the age at the time of saliva collection.

A total of 24 metabolites were detected in the saliva samples: alanine, acetate, butanol, butyrate, choline, citrate, ethanol, formate, fucose, glycine, histidine, isopropanol, lactate, methanol, methylamine, phenylalanine, proline, propane, pyruvate, succinate, taurine, trimethylamine, tyrosine, and 1,2-propanediol. Isopropanol and trimethylamine were found only in saliva samples from SS patients, and only four patients' saliva contained a detectable amount of histidine; therefore, these metabolites were excluded. The total number of metabolites analyzed was thus 21 . The metabolite concentrations in saliva samples collected from pSS patients at the four time points are summarized in Table 1. Both taurine and butyrate differed significantly between the baseline and 1 week $(P=0.014, P=0.028)$, between the baseline and 10 weeks $(P=0.001, P=0.005)$, and between the baseline and 20 weeks $(P<0.001$ and $P=0.005)$. Butanol differed significantly between the baseline and 1 week $(P=0.008)$ and 20 weeks $(P<0.001)$. Furthermore, statistically significant differences between the baseline and 10 weeks were found for choline $(P=0.022)$ and succinate $(P$ $=0.023)$, and between the baseline and 20 weeks for acetate $(P=0.036)$, choline $(P=0.025)$, succinate $(P=0.007)$, ethanol $(P=0.005)$, formate $(\mathrm{P}$ $=0.022)$, propane $(P=0.037)$ and glycine $(P=0.044)$. Other differences in metabolite concentrations were not statistically significant.

Based on the earlier results [12], the most significantly differing (pSS patients vs. controls) metabolites, i.e. choline, taurine, alanine, and glycine, was chosen for in-depth analysis. The variations of these metabolites at the four timepoints are shown in Fig. 1. Compared to the baseline of the healthy controls, choline was significantly elevated in the pSS patients at each time point $(P=0.015,0.023,0.001$, and $<0.001)$. Furthermore, compared to the baseline for the controls, taurine was significantly higher at the three last time points $(P=0.023,0.002$, and 0.001$)$, glycine at the last three time points $(P=0.040,0.032$, and 0.004$)$ and alanine at the last two time points $(P=0.005$ and 0.001$)$.

Inter-individual variation was generally larger among the pSS patients than among the controls (Fig. 1). Significantly enlarged inter-patient variations were observed in the concentrations of choline $(P=0.024,0.033$, 0.024 , respectively for the three time points), alanine $(P=0.028$ at the baseline $)$ and glycine $(P=0.011,0.022,0.021$, and 0.004 , respectively) using Levene's test.

Intra-individual variation in metabolite outputs among pSS patients was lowest for choline (mean coefficient of variation [range]): (31.9\% [16.6-83.5\%]), followed by taurine: (37.3\% [21.5-50.3\%]), glycine: (48.1\% [22.3-99.0\%]), and alanine: (48.5\% [29.0-84.4\%]) (Table 2). Figure 2 shows the intra-individual variations in alanine, glycine, choline, and taurine outputs in the five most representative patients during the follow-up period of 20 weeks. As seen in Table 2, patients \#3, \#5, \#7, and $\# 9$ had experienced SS symptoms for about 10 years and patient $\# 19$ had had symptoms for only three years before diagnosis of SS. Patient \#5 had had serious clinical symptoms and a kidney transplant.

\section{Discussion}

The principal objective of the present study was to assess inter- and intraindividual variation in the salivary metabolic profiles of $\mathrm{pSS}$ patients. To 


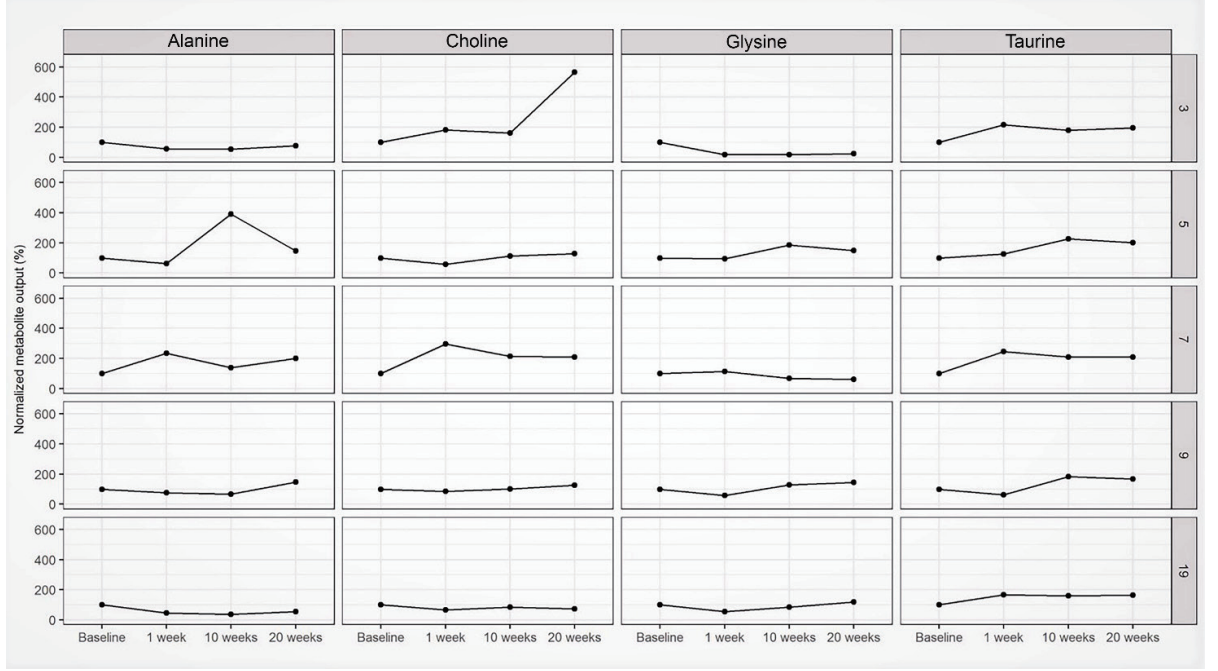

Fig. 2 Intra-individual variations in alanine, glycine, choline and taurine metabolite outputs. Metabolite output ( $\mu \mathrm{mol} / \mathrm{min})$ was derived by multiplying metabolite concentration $(\mu \mathrm{mol} / \mathrm{L})$ by salivary flow rate $(\mathrm{mL} / \mathrm{min})$, and this parameter was normalized by dividing subsequent output values ( 1 week, 10 weeks, 20 weeks) by the baseline output value. The numbers on the right side refer to patient identification codes given at the beginning of the study.

the authors' knowledge, this is the first reported study to have assessed variations in the amounts of specific salivary metabolites, i.e. choline, taurine, glycine, and alanine, using NMR. These metabolites were selected because they differed significantly from the corresponding levels in controls [12] and, based on the existing literature, it was considered that closer examination of these metabolites was warranted to clarify whether they play any potential roles in Sjögren's syndrome.

NMR spectra yield information about the physicochemical condition of salivary metabolites. This study confirmed that it is possible to determine pSS-specific levels of these metabolic components in saliva using quantitative ${ }^{1} \mathrm{H}-\mathrm{NMR}$ spectroscopy. Previous studies of salivary metabolomics have used, for example, various MS methods coupled with separation techniques such as high-performance liquid chromatography, gas chromatography, and ultraperformance liquid chromatography. All of these methods and techniques were aimed at high-quality profiling from a small sample [12]. The main advantages of NMR spectroscopy include its minimal sample handling, unbiased quantification of low-molecular-weight compounds in saliva, and high reproducibility $[8,18,20]$. The consistent quantification of salivary metabolites is due to the inherently reproducible nature of NMR spectroscopy; saliva samples never come into contact with the radiofrequency detector in the NMR spectrometer. This makes NMR metabolomics essentially free of batch effects. Metabolite quantification directly from saliva, without any sample extraction procedures, further contributes to the high reproducibility. A limitation of NMR spectroscopic analysis is that it requires relatively large saliva sample volumes $(\sim 0.5 \mathrm{~mL})$, which may be problematic, especially when collecting unstimulated saliva from patients with dry mouth [21].

To quantify metabolites in absolute terms using NMR spectroscopy, the use of an internal standard of known concentration is required. Consistently with the present study, the majority of previous saliva studies have used TSP as an internal standard [11]. Such a practice is already known to be inappropriate for plasma as TSP binds to albumin, and the bound fraction becomes invisible in the ${ }^{1} \mathrm{H}-\mathrm{NMR}$ spectrum, although it has been observed that the relatively low protein content of saliva may avoid this problem [11]. The accuracy of metabolite quantification using this platform has previously been shown to be comparable to that of biochemical assays [22]. Thus, it can be assumed that any observed differences in metabolite concentrations are due mainly to biological factors.

Saliva gland function may be affected by many conditions and diseases, for example, autoimmune diseases such as SS or may be a secondary effect of rheumatoid disease. Salivary function may be abnormal due to developmental defects, and glands may be damaged due to procedures such as radiation therapy (cancer treatment) [23]. In this study, the salivary choline level was more elevated in SS patients than in the controls at all time points. Choline and its metabolic products are involved in malignant transformation and oxidative stress. For example, choline concentration is elevated in cancer, and several enzymes involved in choline metabolism may be overly expressed [24]. There is evidence that salivary choline levels are also elevated in patients with a history of oral squamous cell carcinoma (OSCC) [25]. SS patients have a much higher risk of developing certain kinds of lymphoma, such as mucosa-associated lymphoid tissue (MALT) lymphoma [6]. However, the levels of other metabolites known to be related to the metabolism of squamous cell carcinoma, including fucose, 1,2-propanediol, and proline $[21,26]$, were not changed in the saliva of pSS patients.

Taurine plays an important role in cellular responses to osmotic stress, regulating volume changes, and the final composition of saliva through sodium flux [27]. In the present study, taurine concentration was elevated in SS patients. However, there seemed to be lower intra-individual variation in patients that had been diagnosed more recently in comparison with those with a long-term SS diagnosis. A higher concentration of taurine in SS patients can mean that there is oxidative stress in the tissues [28]. Choline and taurine may be potential metabolites when searching for SS progression and for monitoring of tissue damage, because choline is linked to cancer metabolism $[6,24,25]$ and taurine is closely involved in oxidative stress $[27,28]$.

Glycine is a very common human metabolite [29], belonging to a family of neurotransmitters and acting as an inhibitor. Glycine plays a role in the synthesis of proteins, and it is known to have immunomodulatory, anti-inflammatory, and cytoprotective properties [29]. Alanine functions as a link between tissues and the liver, working as part of the glucose-alanine cycle. Alanine, via alanine aminotransferase (ALT), is associated with the pathogenesis of diabetes [30]. As the present results show, alanine output varied the most within individuals, and the inter-individual variation in pSS patients was larger than in the controls (Fig. 1). It seems that intraindividual variations in metabolites are greater when patients have had SS for a long time. However, further studies with a larger number of patients and age-matched controls will be needed to confirm this.

Based on identification of 88 metabolites using gas chromatographymass spectrometry, Kageyama et al. [5] have suggested that inflammation of the major salivary glands can affect metabolite profiles, which in turn can be indicative of possible disease phenotypes. Further analysis of more samples using combined NMR/MS analysis might lead to a better understanding of metabolic processes in salivary glands, including patients with SS. Biomarkers for specific forms of SS might allow disease prediction and could be used for monitoring of disease severity. Future studies will need to compare SS and other (autoimmune) disorders to improve the validity of these pSS metabolic biomarkers and their subsequent diagnostic application.

The main limitations of this study were the low number of subjects in the SS and control groups, and the fact that only one saliva sample was collected from each of the controls. The patient population comprised 
only pSS patients, and therefore it remained partly unclear whether the variability of salivary metabolite levels was specifically caused by pSS, as hyposalivation might have been a factor. In the future, patients with other dry mouth conditions such as xerostomia associated with neurogenic disorders or radiation therapy will need to be studied. Therefore, the results of the present study should be confirmed in a larger investigation to verify whether NMR spectroscopy-based salivary metabolomics is appropriate for noninvasive screening and monitoring of pSS. It is obvious that NMR spectroscopy is a technically suitable method for analysis of certain metabolites in saliva and performs better than other methods that are designed to analyze just a single metabolite. NMR spectroscopy represents a reproducible technique for studies of salivary metabolites without any need for complex sample preparation. The use of salivary metabolomics in the field of oral science will open up new avenues in research and clinical practice and provide further insight into pSS, other diseases, and their interrelationships.

\section{Acknowledgments}

This work was partly supported by the Finnish Funding Agency's for Technology and Innovation (Tekes) Oraldent (2186/31/2010) and Oral Cancer $(52 / 31 / 2014)$ projects. MH has received a grant from the Finnish Dental Association Apollonia. Dr Pasi Soininen is thanked for help with the NMR spectroscopy technique and providing valuable advice about NMR.

\section{Conflict of interest}

The authors have no potential conflict of interest to declare.

\section{References}

1. Goules AV, Tzioufas AG (2016) Primary Sjögren's syndrome: clinical phenotypes, outcome and the development of biomarkers. Autoimmun Rev 15, 695-703.

2. Hakala M, Niemelä RK (2000) Does autonomic nervous impairment have a role in pathophysiology of sjögren's syndrome. The Lancet 355, 1032-1033.

3. van der Meulen TA, Harmsen HJM, Bootsma H, Liefers SC, Vich Vila A, Zhernakova A et al. (2018) Dysbiosis of the buccal mucosa microbiome in primary Sjögren's syndrome patients. Rheumatology 57, 2225-2234.

4. Wakamatsu TH, Dogru M, Matsumoto Y, Kojima T, Kaido M, Ibrahim OM et al. (2013) Evaluation of lipid oxidative stress status in Sjögren syndrome patients. Invest Ophthalmol Vis Sci 54, 201-210

5. Kageyama G, Saegusa J, Irino Y, Tanaka S, Tsuda K, Takahashi S et al. (2015) Metabolomics analysis of saliva from patients with primary Sjögren's syndrome. Clin Exp Immuno $182,149-153$.

6. Fragkioudaki S, Mavragani CP, Moutsopoulos HM (2016) Predicting the risk for lymphoma development in Sjogren syndrome: an easy tool for clinical use. Medicine 95 , 3766-3766.

7. García-Villaescusa A, Morales-Tatay J, Monleón-Salvadó D, González-Darder JM, BellotArcis C, Montiel-Company JM et al. (2018) Using NMR in saliva to identify possible biomarkers of glioblastoma and chronic periodontitis. PloS One 13, e0188710.

8. Dame ZT, Aziat F, Mandal R, Krishnamurthy R, Bouatra S, Borzouie S et al. (2015) The human saliva metabolome. Metabolomics 11, 1864-1883.
9. Mikkonen J, Singh S, Herrala M, Lappalainen R, Myllymaa S, Kullaa A (2016) Salivary metabolomics in the diagnosis of oral cancer and periodontal diseases. J Periodont Res 51 , 431-437.

10. Grimaldi M, Palisi A, Rossi G, Stillitano I, Faiella F, Montoro P et al. (2018) Saliva of patients affected by salivary gland tumour: an NMR metabolomics analysis. J Pharm Biomed Anal 160, 436-442.

11. Gardner A, Parkes HG, Carpenter GH, So PW (2018) Developing and standardizing a protocol for quantitative proton nuclear magnetic resonance $\left({ }^{(1)} \mathrm{H}\right.$ NMR) spectroscopy of saliva. J Proteome Res 17, 1521-1531.

12. Mikkonen JJ, Herrala M, Soininen P, Lappalainen R, Tjäderhane L, Seitsalo H et al. (2013) Metabolic profiling of saliva in patients with primary Sjögren's syndrome. Metabolomics: Open Access 3, 128 doi:10.4172/2153-0769.100012

13. De Almeida PDL, Gregio A, Machado M, De Lima A, Azevedo LR (2008) Saliva composition and functions: a comprehensive review. J Contemp Dent Pract 9, 72-80.

14. Vitali C, Bombardieri S, Jonsson R, Moutsopoulos HM, Alexander EL, Carsons SE et al. (2002) Classification criteria for Sjögren's syndrome: a revised version of the European criteria proposed by the American-European consensus group. Ann Rheum Dis 61, 554558.

15. Niemela RK, Takalo R, Paakko E, Suramo I, Paivansalo M, Salo T et al. (2004) Ultrasonography of salivary glands in primary Sjögren's syndrome. A comparison with magnetic resonance imaging and magnetic resonance sialography of parotid glands. Rheumatology (Oxford) 43, 875-879.

16. Seitsalo H, Niemelä RK, Marinescu-Gava M, Vuotila T, Tjäderhane L, Salo T (2007) Effectiveness of low-dose doxycycline (LDD) on clinical symptoms of Sjögren's syndrome: A randomized, double-blind, placebo controlled cross-over study. J Negat Results Biomed 6 , 11

17. Navazesh M (1993) Methods for collecting saliva. Ann N Y Acad Sci 694, 72-77.

18. Silwood CJL, Lynch E, Claxson AWD, Grootveld MC (2002) ${ }^{1} \mathrm{H}$ and ${ }^{13} \mathrm{C}$ NMR spectroscopic analysis of human saliva. J Dent Res 81, 422-427.

19. Aimetti M, Cacciatore S, Graziano A, Tenori L (2012) Metabonomic analysis of saliva reveals generalized chronic periodontitis signature. Metabolomics 8, 465-474.

20. Soininen P, Haarala J, Vepsäläinen J, Niemitz M, Laatikainen R (2005) Strategies for organic impurity quantification by $1 \mathrm{H}$ NMR spectroscopy: constrained total-line-shape fitting. Anal Chim Acta 542, 178-185.

21. Mikkonen, JJ, Singh, SP, Akhi R, Salo T, Lappalainen R, González Arriagada et al. (2018) Potential role of nuclear magnetic resonance spectroscopy to identify salivary metabolite alterations in patients with head and neck cancer. Oncol Lett 16, 6795-6800.

22. Kettunen J, Demirkan A, Wrtz P, Draisma HHM, Haller T, Rawal R et al. (2016) Genomewide study for circulating metabolites identifies 62 loci and reveals novel systemic effects of LPA. Nat Commun 7, 11122.

23. Pedersen AML, Sørensen CE, Proctor GB, Carpenter GH, Ekström J (2018) Salivary secretion in health and disease. J Oral Rehabil 45, 730-746.

24. Glunde K, Bhujwalla ZM, Ronen SM (2011) Choline metabolism in malignant transformation. Nat Rev Cancer 11, 835-848.

25. Ohshima M, Sugahara K, Kasahara K, Katakura A (2017) Metabolomic analysis of the saliva of japanese patients with oral squamous cell carcinoma. Oncol Rep 37, 2727-2734.

26. Wang Q, Gao P, Wang X, Duan Y (2014) Investigation and identification of potential biomarkers in human saliva for the early diagnosis of oral squamous cell carcinoma. Clin Chim Acta 427, 79-85

27. Mozaffari MS, Borke JL (2002) Taurine in submandibular gland of the rat: effect of muscarinic drugs. J Histochem Cytochem 50, 527-532.

28. Porto-Mascarenhas EC, Assad DX, Chardin H, Gozal D, De Luca Canto G, Acevedo AC et al. (2017) Salivary biomarkers in the diagnosis of breast cancer: a review. Crit Rev Oncol Hematol 110, 62-73.

29. Pérez-Torres I, Zuniga-Munoz AM, Guarner-Lans V (2017) Beneficial effects of the amino acid glycine. Mini Rev Med Chem 17, 15-32

30. Malicka B, Skoskiewicz-Malinowska K, Kaczmarek U (2016) Salivary lactate dehydrogenase and aminotransferases in diabetic patients. Medicine 95, e5211. 